\title{
Evaluación de la eficacia de WhatsApp en un programa grupal de reducción de daños asociados al consumo inyectado de drogas
}

\section{Evaluation of the efficacy of WhatsApp through a harm reduction intervention group for injecting drug users}

\author{
Fran Calvo*,**, Oriol Turró-Garriga***, Xavier Carbonell ****. \\ * Departament de Pedagogia, Institut de Recerca sobre Qualitat de Vida, Universitat de Girona. \\ ** Grupo de Investigación en Salud Mental y Adicciones, Instituto de Investigación Biomédica de Girona [IdIBGi], \\ Institut d'Assistència Sanitària, Girona. \\ *** Grupo de Investigación en Envejecimiento, Discapacidad y Salud [IdIBGi], Girona. \\ **** FPCEE Blanquerna, Universitat Ramon Llull, Barcelona.
}

\section{Resumen}

El presente estudio analiza el uso de una aplicación de mensajería instantánea (WhatsApp ${ }^{\circledR}$ ) como canal de acceso a personas que se inyectan drogas. Se diseñó un estudio observacional longitudinal prospectivo de ocho semanas y tres observaciones en cinco centros de adicciones en Cataluña. Participaron 105 personas que consumían drogas por vía parenteral, distribuidas en cinco grupos de intervención grupal. Se compararon los resultados de la escala Risk Assessment Battery (RAB) (después de ser traducida al español y analizada su consistencia interna) en las tres fases de análisis pre test, post intervención y un mes después de la intervención. Los resultados indican una disminución significativa de las puntuaciones RAB tras la intervención a través de WhatsApp ${ }^{\circledR}$. Se concluye que la intervención grupal a través de WhatsApp presenta grandes potencialidades para realizar intervenciones en reducción de daños y reducir el riesgo de contagio del VIH.

Palabras clave: Programa de intercambio de jeringuillas; drogas en la calle; dependencia de drogas; eSalud; reducción de daños; personas sin-hogar; WhatsApp ${ }^{\circledR}$; mensajería instantánea; redes sociales en línea.

\begin{abstract}
This study aims to analyse the use of an instant messaging app (WhatsApp ${ }^{\circledR}$ ) as a means of communication for reaching people who inject drugs. An eight-week prospective longitudinal and observational study with three observations was designed for five addiction centres in Catalonia. The participants were 105 people who inject drugs, distributed in five intervention groups. The results of the Risk Assessment Battery (RAB) were compared in the three levels of analysis pre-test, post intervention and one month after the intervention. The main results indicate a significative reduction in RAB scores after the intervention. The main conclusion was that the WhatsApp ${ }^{\circledR}$ intervention has great potential for developing harm reduction interventions and to reduce the HIV contagion risk.

Keywords: Needle exchange program; street drugs; drug dependence; eHealth; harm reduction; homeless persons; WhatsApp ${ }^{\circledR}$; online social networking.
\end{abstract}




\section{Introducción}

$\mathrm{E}$ 1 concepto reducción de daños (REDAN) hace referencia a aquellas intervenciones, programas y políticas cuyo objetivo es minimizar los efectos nocivos del consumo de drogas (Martínez-Luna et al., 2018; Mira, Llinás, Lorenzo y Aibar, 2009). Es uno de los cuatro ejes de la atención a las drogodependencias junto con las políticas de control de la oferta, la prevención y el tratamiento de las conductas adictivas. Son una alternativa a los modelos de atención especializada más exigentes y basados en la abstinencia, cuyos compromisos no pueden ser cumplidos por determinados pacientes y priorizan la retención de las personas que consumen drogas por vía parenteral (PCDVP) en los centros de salud (Erickson, 1995).

En España, la REDAN ha conseguido disminuir la mortalidad de las PCDVP asociada a los problemas del consumo inyectado de heroína, al aumento de infecciones del Virus de la Inmunodeficiencia Humana (VIH) y a la mortalidad asociada al Síndrome de la Inmunodeficiencia Adquirida (SIDA) (Barrio et al., 2012). Pese a este impacto positivo, las PCDVP del estado español presentan las tasas de infección de VIH y de Virus de la Hepatitis C (VHC) de las más elevadas de Europa Occidental (30,6\% y 79,6\% respectivamente) (Stone, 2014 y 2016). Aún lejos de las dramáticas cifras de mortalidad asociada al VIH entre los jóvenes de mediados de los años noventa, en la actualidad un $15,4 \%$ de PCDVP pierden la vida como consecuencia de la infección de VIH y VHC, siendo uno de los principales factores de riesgo su coinfección (Lozano, Domeque, Perálvarez, Torrellas y Gonzalo, 2019). Las prácticas de riesgo relacionadas con la inyección de drogas, sean directas (compartir jeringuillas usadas) o indirectas (compartir parafernalia de inyección como filtros, recipientes, agua o cargar en las jeringuillas dosis disueltas compartidas) son altas, especialmente en población joven (Folch et al., 2016). La exposición a factores de riesgo de contagio por vía sexual entre PCDVP es muy elevada y un $34 \%$ mantiene relaciones sexuales sin protección esgrimiendo conocer y asumir los riesgos con motivo de un displacer asociado al uso del preservativo (Calvo-García, Turró-Garriga y Giralt-Vázquez, 2014). La exclusión social extrema y el sinhogarismo se posicionan como uno de los principales factores de riesgo de inicio de consumo inyectado entre los jóvenes (Calvo, Carbonell y Badia, 2018; Folch et al., 2016). En consecuencia, numerosos autores plantean tanto la necesidad de revitalizar los programas REDAN como la de incorporar nuevos enfoques que complementen los que ya están en funcionamiento (Bosque-Prous y Brugal, 2016; Fuente et al., 2006; Trujols et al., 2010).

Uno de los cambios más significativos en la organización de la atención a la salud en los últimos años es su progresiva incorporación de las Tecnologías de la Información y la Comunicación (TIC). La eSalud (eHealth), definida como el uso de las TIC para el desarrollo de la salud y la
mSalud (mHealth), definida como la atención de la salud apoyada por la incorporación de dispositivos móviles como teléfonos inteligentes, tabletas y otros dispositivos, son parte creciente de la oferta de salud de los países miembros de la Unión Europea (World Health Organization, 2011)

La eSalud y la mSalud incluyen las Redes Sociales Online (RSO) al ser utilidades basadas en Internet que posibilitan la creación e intercambio de contenidos multimedia generados por los propios usuarios (Kaplan y Haenlein, 2010). Esta aplicabilidad en salud se basa en la filosofía del web 2.0 cuyo principal potencial es su contribución a modelos de salud colaborativa y abierta que ofrecen al paciente más capacidad para manejar su propio proceso, empoderándolo en relación a su salud (Armayones et al., 2015). El uso de las RSO en eSalud ha demostrado ser efectivo para la promoción del uso del preservativo en personas en situación de sinhogarismo consumidoras de drogas (Rice, 2010), para la reducción de daños directos relacionados con el consumo de alcohol y otras drogas (Rice, Milburn y Monro, 2011) y para facilitar la aceptación de los programas de reducción de daños y de prevención (Rice, Tulbert, Cederbaum, Barman Adhikari y Milburn, 2012). Las intervenciones psicoeducativas dirigidas al aprendizaje de su uso por las personas en situación de exclusión social presentan ya beneficios psicológicos en sí mismos (Calvo y Carbonell, 2018).

Teniendo en cuenta la importancia de las intervenciones psicosociales para mejorar la calidad de vida de los pacientes consumidores de drogas por vía parenteral (Fernández, González, Saiz, Gutiérrez y Bobes, 1999), esta investigación pretende analizar las potencialidades de utilidad asociadas al uso de un servicio móvil de mensajería instantánea (WhatsApp ${ }^{\circledR)}$ como canal de comunicación complementario en la organización de grupos de discusión grupales para la reducción de daños.

\section{Método}

\section{Diseño}

Estudio observacional longitudinal prospectivo.

\section{Población objeto de estudio}

Personas con consumo activo de drogas por vía parenteral atendidas en centros de reducción de daños, centros de tratamiento de las adicciones o centros específicos para personas sin-hogar.

\section{Muestra}

Muestra de conveniencia procedente de cinco centros (dos centros de tratamiento de las adicciones, dos centros de reducción de daños y un centro de atención a personas sin-hogar). De acuerdo con el número estimado de PCDVP atendidas en los cinco centros participantes $(n=300)$ y asumiendo el principio de máxima indeterminación, $\mathrm{p}=$ 
$q=50$, con un margen de error del $8 \%$ y un nivel de confianza del 95\%, se determinó un tamaño muestral de 101 participantes.

Los criterios de inclusión de los participantes fueron haber consumido drogas inyectadas el último año y disponer de un teléfono inteligente. En el reclutamiento de participantes, los referentes de cada uno de los centros preguntaron a los posibles candidatos por su interés en participar. En caso de cumplir los criterios de inclusión se anotaron en un listado y en el momento del inicio de la intervención se ratificó su interés y si aún cumplían con dichos criterios de inclusión. A continuación, recibieron por escrito un impreso informativo sobre los objetivos del estudio, su metodología y posibles resultados donde se explicitaba su derecho a abandonarlo en cualquier momento. Posteriormente firmaron el consentimiento informado. Se consideró como criterio de exclusión la manifestación expresa de no desear continuar en el estudio, el abandono voluntario del grupo de WhatsApp ${ }^{\circledR}$ y la negativa a volver a cumplimentar en test en alguna de las tres observaciones.

\section{Procedimiento}

Tras analizar el uso de dispositivos móviles y teléfonos inteligentes de personas en situación de exclusión social extrema y consumo de drogas inyectadas (Calvo, Carbonell, Turró y Giralt, 2018; Genz et al., 2015), se diseñó una intervención grupal de ocho semanas, con el objetivo de reducir el impacto de los daños asociados al consumo de drogas inyectadas basada en la Terapia de Reducción de Daños [Harm Reduction Therapy], que prioriza la retención en el programa de intervención como principal elemento para facilitar el cambio (Little, Hodari, Lavender y Berg, 2008). Los participantes se distribuyeron en siete grupos de WhatsApp con el objetivo de facilitar la discusión, tal y como sucede en los grupos presenciales. La intervención fue precedida de una propuesta de temática semanal basada en alguno de los temas más relevantes en materia de reducción del riesgo de infección del VIH. En los grupos de WhatsApp, los participantes interaccionaban entre ellos o se dirigían directamente a los profesionales haciendo preguntas, sugerencias, explicando experiencias, aclarándose dudas entre ellos, e interaccionando. Las intervenciones de los investigadores fueron mínimas para tratar que el propio grupo mediara en la respuesta de las dudas y aprovechar las potencialidades descritas sobre el apoyo entre iguales del grupo de discusión, siguiendo el procedimiento habitual de gestión de grupos de este tipo. La intervención se diseñó en forma de grupo de discusión cuya efectividad ha sido ampliamente demostrada (Calvo, Pérez, Sacristán y Paricio, 2009; Cheung et al., 2015). Un análisis en profundidad de estos contenidos y la propuesta de metodología de intervención grupal ya han sido descritas previamente (Calvo, Carbonell, Giralt, Lloberas y Turró, 2017).
La investigación fue aprobada por el Comité de Ética de Investigación CEI-Girona con código XSO_2017, el 7 de junio de 2017.

\section{Variables del estudio e instrumentos de evaluación}

Se usó un cuestionario ad hoc para conocer edad, sexo, infección por VIH, condición de sinhogarismo, estar o no en un programa de mantenimiento con Metadona -PMMy droga inyectada principal en el último mes.

Variable dependiente: Los datos sobre tipología de consumo de drogas y prácticas de riesgo de contagio de VIH se obtuvieron con la escala Risk Assessment Battery (RAB) (Navaline et al., 1994). Consta de 29 ítems, 17 de ellos puntuables, cuyo valor se comprende entre 0 y 40 puntos (cociente $0-1)$. El resto de ítems proporcionan información descriptiva sobre el consumo de drogas, la actividad sexual, el nivel de preocupación sobre la posibilidad de contagio del VIH y el conocimiento del paciente de su última analítica realizada y su estado serológico. La escala ha mostrado una consistencia interna de 0,82 en su versión estándar y de 0,86 en su versión electrónica (Navaline et al., 1994). La escala fue sometida a un proceso de traducción y retrotraducción al castellano y adaptada a población española con una consistencia interna de 0,81 calculada sobre el total de observaciones de los participantes (105 participantes x 3 observaciones $=315)$. La escala original no presenta datos específicos de estructura factorial, puntos de corte ni de sensibilidad y especificidad. En el Anexo 1 se puede consultar la escala tal como se usó en la investigación.

Finalmente, como información cualitativa adicional, al finalizar las ocho semanas de intervención, se planteó a los participantes la posibilidad de responder a tres preguntas abiertas de valoración de la intervención: i) valoración sobre la información propuesta en los grupos de intervención; ii) valoración sobre el canal WhatsApp como canal comunicativo con personas que consumen drogas inyectadas; y iii) valoración del grupo como parte del proceso terapéutico o de reducción de daños que seguían en el momento de realizar el programa.

\section{Análisis estadístico}

Para la descripción de los datos se usaron medidas de tendencia central y dispersión y análisis de frecuencias absolutas y relativas para los datos cuantitativos y cualitativos respectivamente. Se usaron correlaciones de Pearson para la comparación de variables cuantitativas, y estadísticos de comparación de medias de las variables cuantitativas entre grupos según criterios de normalidad y tablas de contingencia para la comparación de variables cualitativas. Las observaciones se llevaron a cabo al inicio de la formación $\left(\mathrm{T}^{1}\right)$, a su finalización $\left(\mathrm{T}^{2}\right)$ y un mes después $\left(\mathrm{T}^{3}\right)$. Su usó la t de Student para muestras relacionadas para analizar la diferencia de medias en las puntuaciones intra-grupos. Se calculó el índice de diferencias de puntuaciones intra-gru- 
pos entre $\mathrm{T}^{1}$ y $\mathrm{T}^{3}[(T 1-T 3 / T 1) * 100]$ y se usó como variable dependiente en el modelo de regresión lineal ajustado con el objetivo de determinar las variables asociadas a la mayor diferencia. Se ajustó un modelo de regresión lineal mixto para determinar si la diferencia en la puntuación no era atribuible al centro o las variables específicas individuales.

\section{Resultados}

\section{Descripción de la muestra}

Se reclutaron un total de 130 PCDVP de las que 11 fueron excluidas por carecer de teléfono inteligente y 14 abandonaron el grupo de WhatsApp; por lo tanto, la muestra final la constituyeron 105 PCDVP.

El 86,7\% de los participantes fueron hombres, con una media de edad de 41,3 años $(\mathrm{DE}=6,7)$. Todos los participantes habían consumido alguna droga durante el mes previo a la intervención, aunque refirieron no haber consumido opioides (excepto metadona), anfetaminas, metanfetamina o alucinógenos. El 32,4\% de la muestra refirieron estar contagiados de VIH $(\mathrm{n}=34)$. De los 71 participantes que desconocían si estaban contagiados, el 88,7\% ( $\mathrm{n}=63$ ) refirieron algún nivel de preocupación sobre la posibilidad de estarlo y un 97,2\% ( $\mathrm{n}=69)$ sobre la posibilidad de haber estado expuesto al virus. El 76,1\% de los participantes ( $\mathrm{n}=54)$ refirieron haber realizado una analítica de sangre para determinar la presencia de VIH una media de 1,9 ocasiones $(\mathrm{DE}=2,8)$ y de 1,7 años antes del pre-test (DE $=1,7)$. El 88,6\% de los participantes estaban incluidos en un PMM.

El 39,6\% de los participantes manifestaron estar en situación de sinhogarismo. Se encontraron diferencias entre las personas sin-hogar y las que no lo eran, especialmente en el tipo de consumo realizado el último mes. Como se observa en la Tabla 1, las personas sin-hogar presentaron mayores índices de consumo de cannabis ( $100 \%$ vs. $80,6 \%)$, alcohol $(100 \%$ vs. $89,5 \%)$ y cocaína $(81,6 \%$ vs. $55,2 \%)$, especialmente inyectada $73,7 \%$ vs. $66,1 \%$ ) y menor consumo de heroína inyectada $(42,1 \%$ vs. $88,1 \%)$ y speedball inyectado $(21,1 \%$ vs. $58,2 \%)$. Las personas sin-hogar presentaron una mayor adscripción al PMM (94,7\% vs. 82,1\%).

Tabla 1. Descriptivos de la muestra en el pretest.

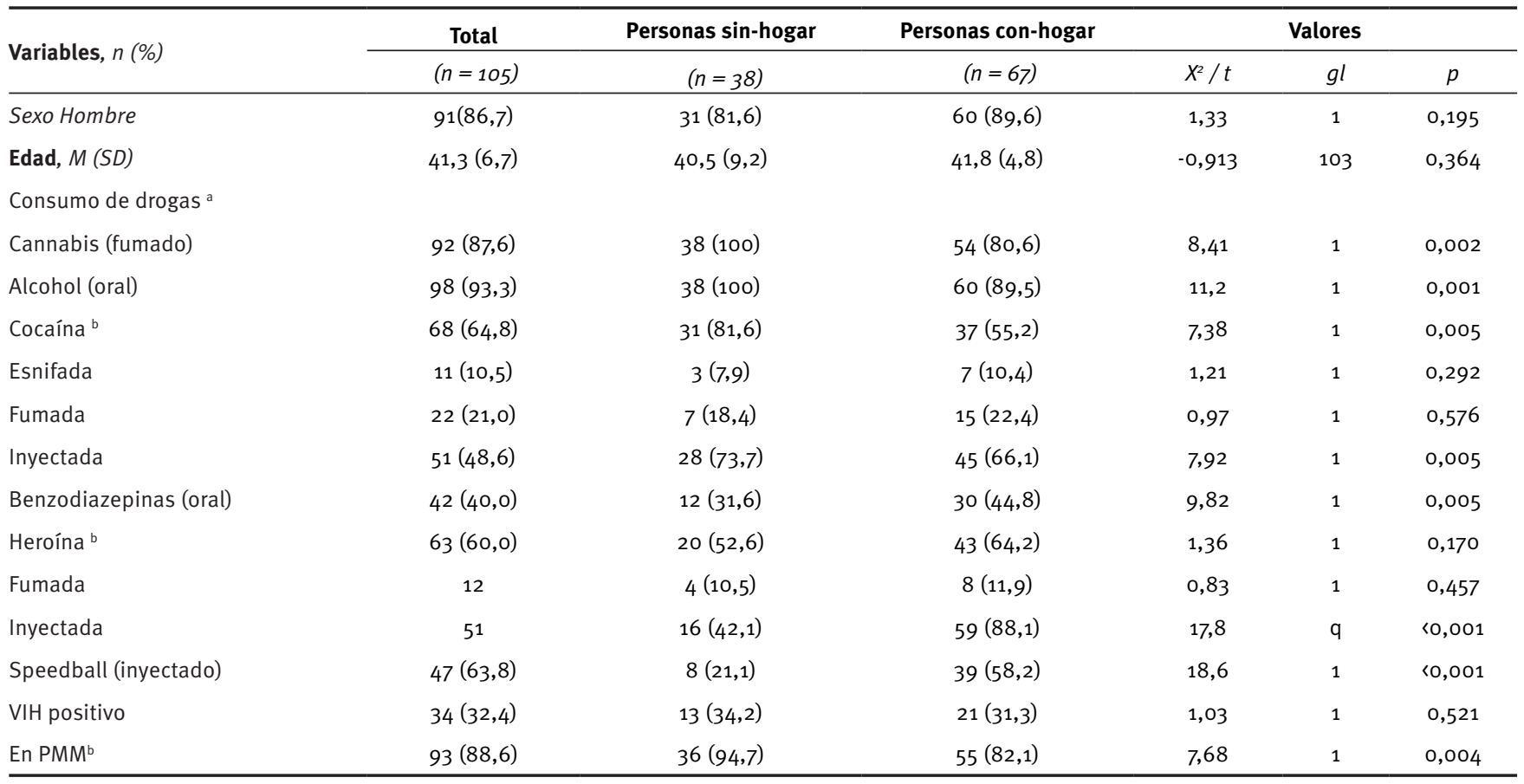

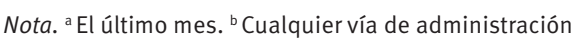

\section{Comparación de las medidas intra-grupo}

La media de puntuaciones de la RAB en la $\mathrm{T}^{1}$ fue de 13,35 $(\mathrm{De}=5,42)$ que disminuyó hasta 9,49 $(\mathrm{DE}=5,58)$ en la $\mathrm{T}^{2}$, con una disminución de la media de puntuaciones de 3,87 puntos $(\mathrm{DE}=7,89 ; \mathrm{t}=5,021 ; \mathrm{gl}=104 ; \mathrm{p}<0,001)$. En la medida de la $\mathrm{T}^{3}$ la media de puntuaciones fue de $8,70(\mathrm{DE}=5,01)$ que, pese a no presentar una disminución significativa con respecto a la $\mathrm{T}^{2}(\mathrm{M}=0,79 ; \mathrm{DE}=4,23 ; \mathrm{t}=$ 1,915; gl = 104; $\mathrm{p}=0,058)$, sí que indica una tendencia a la significación estadística. La diferencia de medias de puntuaciones entre la $\mathrm{T}^{1}-\mathrm{T}^{3}$ fue de 4,65 puntos $(\mathrm{DE}=7,25 ; \mathrm{t}=$ $6,58 ; \mathrm{gl}=104 ; \mathrm{p}<0,001)$ (Figura 1). Se pueden observar los ítems puntuables dela RAB en la Tabla 2. 
Tabla 2. Descripción ítems puntuables de la escala.

\begin{tabular}{|c|c|c|c|c|c|c|c|}
\hline \# & Item & Rango & Me & $\mathbf{P M}^{\mathrm{a}}(\%)$ & M & DE & $\mathbf{r}^{\mathbf{b}}$ \\
\hline 1 & ¿Se ha inyectado drogas durante los últimos 6 meses? & $0-1$ & 1 & 82,6 & 851 &, 319 &,- 917 \\
\hline 2 & $\begin{array}{l}\text { ¿Ha compartido jeringuillas o algún material de inyección (cazoletas u otros recipientes, filtro, } \\
\text { algodón, etcétera durante los últimos } 6 \text { meses? }\end{array}$ & $0-3$ & 0 & 23,6 &, 623 & 1,11 & $672^{\star \star}$ \\
\hline 3 & ¿Con cuánta gente diferente ha compartido jeringuillas los últimos 6 meses? & $0-3$ & 0 & 2,0 & ,346 & ,675 & ,691* \\
\hline 8 & $\begin{array}{l}\text { En los últimos } 6 \text { meses, ¿con qué frecuencia ha estado en un chutadero, casa ocupada u otro lugar } \\
\text { donde la gente acostumbra a ir para inyectarse drogas? }\end{array}$ & $0-3$ & 0 & 31,6 & 1,23 & 1,42 &, $532^{\star \star}$ \\
\hline 12 & $\begin{array}{l}\text { ¿Con qué frecuencia ha compartido agua estéril (el agua que viene en el kit de intercambio de } \\
\text { jeringuillas) durante los últimos } 6 \text { meses? }\end{array}$ & $0-3$ & 0 & 8,6 & ,639 & ,901 &, $421^{*}$ \\
\hline 13 & $\begin{array}{l}\text { ¿Con qué frecuencia ha compartido cazoletas u otro recipiente para preparar las dosis, durante los } \\
\text { últimos } 6 \text { meses? }\end{array}$ & $0-3$ & 0 & 5,1 & ,498 & ,873 &, $396^{\star *}$ \\
\hline 14 & $\begin{array}{l}\text { ¿Con qué frecuencia ha compartido filtros o algodones para filtrar las dosis, durante los últimos } 6 \\
\text { meses? }\end{array}$ & $0-3$ & 0 & 4,8 &, 563 & ,764 &, $321^{\star \star}$ \\
\hline 15 & $\begin{array}{l}\text { En los últimos } 6 \text { meses, ¿con qué frecuencia ha dividido dosis con otras personas usando una } \\
\text { jeringuilla para introducir o cargar a/desde otra jeringuilla dicha dosis (backfront loading, por } \\
\text { ejemplo)? }\end{array}$ & $0-3$ & 0 & 1,1 & 981 & ,894 &, $411^{\star \star}$ \\
\hline 16 & ¿Cómo se describiría a usted mismo? (Heterosexual, Gay/Lesbiana, Bisexual) & $0-3$ & 1 & 6,4 & 2,01 &, 512 &,- 921 \\
\hline 17 & ¿Con cuántos hombres ha tenido relaciones sexuales durante los últimos 6 meses? & $0-3$ & 0 & 3,0 & ,609 & ,743 &,$- 235^{*}$ \\
\hline 18 & ¿Con cuantas mujeres ha tenido relaciones sexuales durante los últimos 6 meses? & $0-3$ & 1 & 22,8 & 1,12 & ,661 &, $402^{\star *}$ \\
\hline 19 & $\begin{array}{l}\text { En los últimos } 6 \text { meses, ¿con qué frecuencia ha mantenido relaciones sexuales para conseguir } \\
\text { drogas? }\end{array}$ & $0-3$ & 0 & 22,6 & ,574 & ,802 & ,097 \\
\hline 20 & $\begin{array}{l}\text { En los últimos } 6 \text { meses, ¿con qué frecuencia le ha dado drogas a otra persona para poder tener sexo } \\
\text { con él/ella? }\end{array}$ & $0-3$ & 1 & 0,6 & ,971 & ,888 &, $472^{\star \star}$ \\
\hline 21 & En los últimos 6 meses, ¿Con qué frecuencia pagó dinero para tener sexo con alguien? & $0-3$ & 0 & 11,7 &, 406 & ,645 & ,112 \\
\hline 22 & $\begin{array}{l}\text { En los últimos } 6 \text { meses, ¿con qué frecuencia le dio dinero a alguien con el objetivo de tener sexo con } \\
\text { ellos/ellas? }\end{array}$ & $0-3$ & 1 & 2,4 & ,914 & ,886 &, $504^{\star \star}$ \\
\hline 24 & $\begin{array}{l}\text { En los últimos } 6 \text { meses, ¿con qué frecuencia ha usado preservativo para mantener relaciones } \\
\text { sexuales? }\end{array}$ & $0-3$ & 1 & 1,8 & ,818 & 898 & ,432** \\
\hline
\end{tabular}

Nota. ${ }^{a}$ Porcentaje de puntuación máxima. ${ }^{b}$ Correlación con respecto el total. *p<,05. ${ }^{\star \star} p<, 001$.

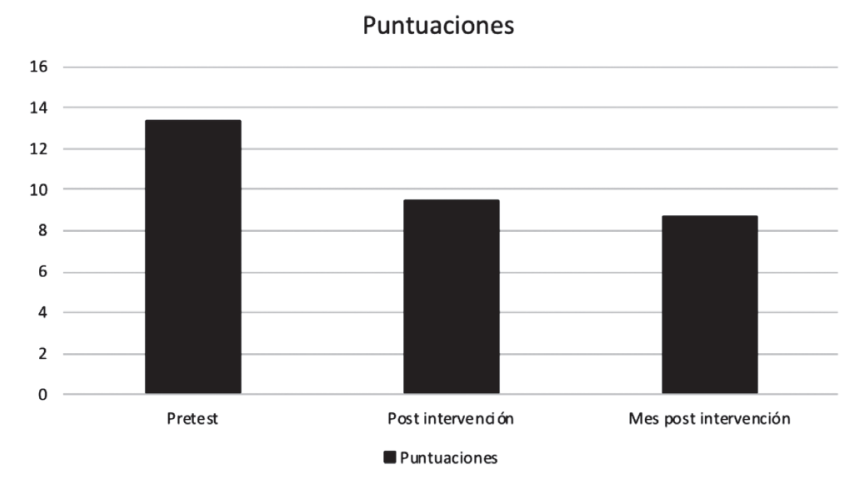

Figura 1. Diferencias de medias de las puntuaciones de la escala Risk Assessment Battery durante los tres momentos de la intervención.

\section{Lineal mixto}

El análisis lineal mixto para medidas repetidas mostró el efecto de la intervención sobre la $\mathrm{RAB}(\mathrm{F}=28,5$; df=2; $\mathrm{p}<0,001$ ). Ajustado por las variables pre-test (sociodemográficas y clínicas) se observaron diferencias significativas entre T3 y T1 pero no entre T3 y T2 (Tabla 3). A partir de la matriz de varianzas y la estimación de parámetros de covarianza, se obtuvo que solamente el $21,9 \%$ de la varianza en la puntuación de la RAB es atribuible a la diferencia entre sujetos mientras que el $78,1 \%$ a la intervención.

\section{Participación en los grupos de WhatsApp ®}

Los participantes se distribuyeron en siete grupos de WhatsApp ${ }^{\circledR}$ con una media de 15,1 participantes por grupo $(\mathrm{DE}=1,8)$. Una vez iniciada la situación experimental, los participantes realizaron un total de 21.893 intervenciones en forma de mensajes de texto, símbolos no textuales (emoticonos), videos o audios entre el $\mathrm{T}^{1}$ y el $\mathrm{T}^{2}$.

Estas intervenciones se corresponden con una media de $3.127,6(\mathrm{DE}=752,1)$ intervenciones por grupo; una media de 391,0 $(\mathrm{DE}=121,8)$ intervenciones por grupo y semana y una media de 48,9 (DE = 31,9) intervenciones diarias por grupo. La media de intervenciones por persona durante las ocho semanas de duración del programa fue de 208,5 $(\mathrm{DE}=214,6)$ intervenciones.

Del total de intervenciones, el 54,7\% ( $\mathrm{n}=11.986$ ) fueron expresiones de dudas o preguntas propias de la reducción de daños. De estas preguntas, el 38,4\% ( $\mathrm{n}=4.597$ ) fueron sobre los principales riesgos de sobredosis, el 16,5\% $(\mathrm{n}=1.981)$ sobre el procedimiento para acceder a una analítica rápida de VIH o VHC, el 10,2\% ( $\mathrm{n}=1.224)$ sobre 
Tabla 3. Modelo de regresión lineal ajustado para diferencias intragrupo. Variable dependiente resultado escala Risk Assessment Battery.

\begin{tabular}{lcccccccc}
\hline \multirow{2}{*}{ Parámetro } & \multirow{2}{*}{ Estimación } & \multirow{2}{*}{ Error Est. } & \multirow{2}{*}{ gl } & t & Sig. & \multicolumn{2}{c}{ IC 95\% } \\
\cline { 6 - 8 } & & & & & & Min. & Máx. \\
\hline Intercep. & 8,70 & 0,52 & 284,86 & 16,78 & $<0,001$ & 7,68 & 9,73 \\
Tiempo $^{1}$ & 4,57 & 0,65 & 208 & 7,05 & $<0,001$ & 3,29 & 5,85 \\
Tiempo $^{2}$ & 0,76 & 0,65 & 208 & 1,17 & 0,241 & $-0,52$ & 2,04 \\
Tiempo $^{3}$ & $0^{*}$ & 0 & & & & & \\
\hline
\end{tabular}

Nota. ${ }^{*}$ Este parámetro es cero porque es redundante.

acceso a material de inyección, el 9,9\% $(\mathrm{n}=1.181)$ sobre interacción de drogas, el 9,5\% ( $\mathrm{n}=1.136)$ sobre niveles de pureza de las drogas, el 8,1\% ( $\mathrm{n}=976)$ sobre acceso a servicios sociales (prestaciones, servicios de pernocta o alimentación) y el 7,4\% (891) sobre acceso a tratamiento o tipos de tratamiento de la adicción.

Según estos datos sobre preguntas o dudas, se formuló una media de $1.712,3(\mathrm{DE}=471,4)$ preguntas por grupo, con una media de $214,1(\mathrm{DE}=264,1)$ preguntas por semana y grupo, y 53,5 $(\mathrm{DE}=31,9)$ preguntas o dudas por día y grupo.

De las 9.907 intervenciones de los participantes que no fueron expresión de dudas o preguntas, un 24,4\% ( $\mathrm{n}=2.421)$ se correspondieron con respuestas a las preguntas realizadas por otros participantes, un $12,1 \%$ ( $\mathrm{n}=$ 1196) fueron mensajes de apoyo hacia otros participantes, un $9,9 \%$ (977) fueron aseveraciones a informaciones que proporcionaron los gestores de los grupos. Finalmente, un $53,6 \%(n=5.313)$ de las intervenciones se corresponden a mensajes sin contenido en si mismo y que forman parte de la interacción de la conversación (mensajes de asentimiento, énfasis en muchas ocasiones en forma de emoticonos o el uso de los signos de puntuación o acrónimos propios del lenguaje textual virtual). Ver Figura 2

Los gestores de los grupos intervinieron un total de 2.431 ocasiones: Un 4,3\% ( $\mathrm{n}=104)$ para proponer los contenidos de las temáticas a trabajar en las discusiones, un $56,3 \%(\mathrm{n}=1.369)$ para responder a dudas o preguntas sobre los contenidos propuestos y que los propios usuarios no daban respuesta por sí mismos en el proceso de discusión, un 24,1\% ( $\mathrm{n}=587)$ para dinamizar los grupos y un $15,3 \%(\mathrm{n}=371)$ para reconducir intervenciones no adecuadas (preguntas personales entre participantes que no tenían que ver con la discusión, bromas, contenidos audiovisuales no apropiados como chistes, etcétera).

Por último, las respuestas abiertas sobre su experiencia en los grupos de WhatsApp fueron codificadas y clasificadas para permitir su análisis. Sobre los contenidos de los grupos, 102 participantes refirieron que eran adecuados y que respondieron a dudas previas o ampliaron la información que tenían sobre los temas tratados, 81 participantes destacaron las posibilidades de inmediatez para acceder a la información y a las respuestas tanto de los iguales como de los gestores del grupo y 51 subrayaron que el espacio virtual podía ser un complemento a las prestaciones habituales de los servicios de tratamiento y de reducción de daños a los que están adscritos: 32 debido a la superación de las barreras de acceso tales como horarios establecidos, ya que al grupo virtual podían acceder cuando quisieran y 19 debido a que el profesional del grupo de WhatsApp respondía rápidamente a las demandas del grupo. Todos los participantes que acabaron el proceso estarían dispuestos a participar en grupos virtuales de forma periódica o continuada como parte de su proceso terapéutico.

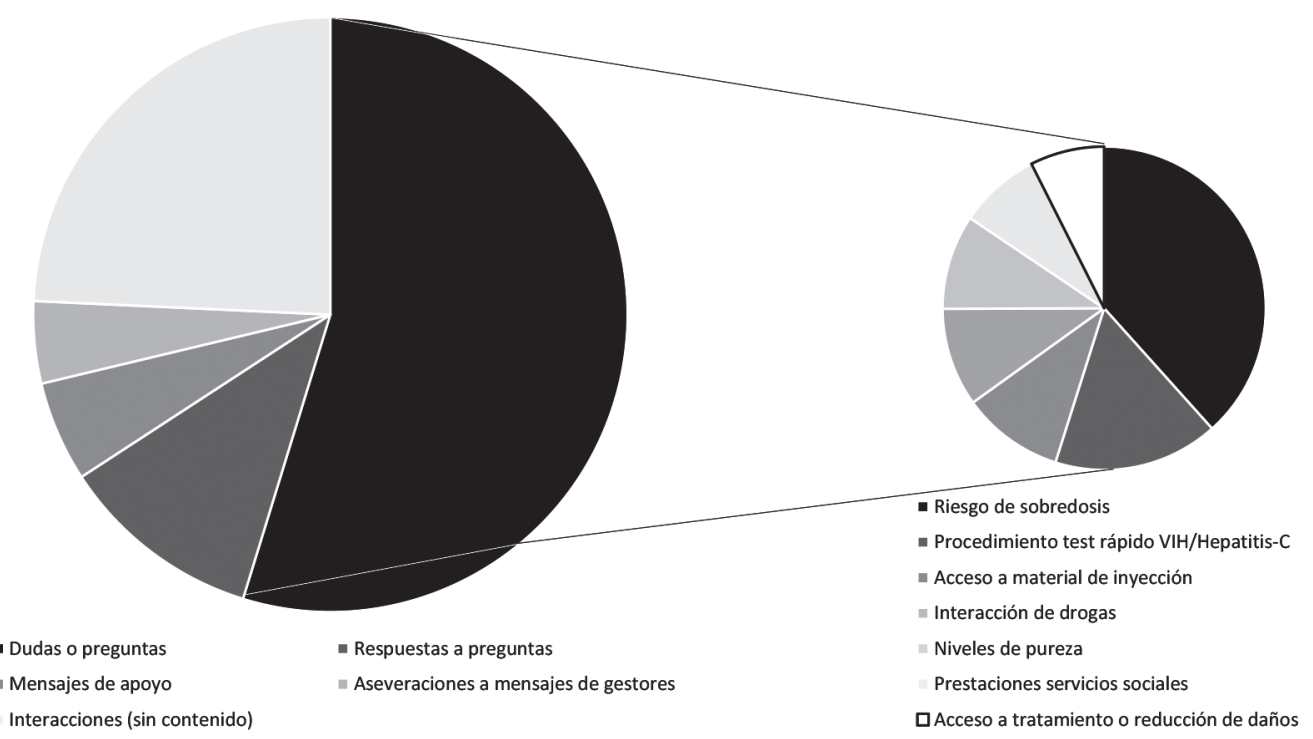

Figura 2. Contenido de las intervenciones en los diferentes grupos de WhatsApp®. 


\section{Discusión}

El objetivo de este estudio fue comprobar la viabilidad en el contexto clínico del uso de un servicio de mensajería instantánea para reducir el riego de infección de VIH asociado al consumo de drogas por vía parenteral. Para ello se diseñó un estudio multicéntrico longitudinal de tres observaciones pretest, post intervención y un mes después de la intervención. Se escogió la RAB, una escala auto-administrada que mide la participación en actividades que aumentan la probabilidad de contraer el VIH, en ausencia de escalas específicas de reducción de daños en castellano. De entre las diferentes escalas en lengua inglesa, la opción de la $\mathrm{RAB}$ respondió a que evalúa un tipo de paciente de difícil retención, se ajustaba a las demandas ordinarias de la historia clínica de los servicios públicos de drogodependencias donde se llevó a cabo el estudio, y es confidencial sobre las prácticas de intercambio de material de inyección y la actividad sexual asociada con el riesgo de contagio entre personas que consumen drogas inyectadas (National Institute on Drug Abuse, 2018).

Se pueden extraer dos enseñanzas principales de esta investigación. La primera es la viabilidad del uso de las RSO para este tipo de intervenciones. La disminución de las puntuaciones de la escala $\mathrm{RAB}$ entre $\mathrm{T}^{1} \mathrm{y} \mathrm{T}^{3}$ sugiere un menor riesgo potencial de infección VIH (National Institute on Drug Abuse, 2018). La segunda tiene que ver con la capacidad de retención de los participantes. La atención ambulatoria en drogodependencias se ve afectada por un elevado índice de abandono (Martínez-González, Albein-Urios, Lozano-Rojas y Verdejo-García, 2014). De hecho, más allá de la retención producida por la toma de sustitutivos opioides, no se han encontrado terapias conductuales y educativas, de asesoramiento o de apoyo que faciliten la retención (Timko, Schultz, Cucciare, Vittorio y Garrison-Diehn, 2016). En este estudio, los grupos de intervención presentaron una capacidad de adherencia elevada y superior a otras intervenciones terapéuticas en adicciones (Calvo et al., 2018), reduciendo las limitaciones inherentes a los servicios presenciales como horarios, listas de espera, gasto en desplazamientos, organización personal, etcétera, tal y como sugirieron los propios participantes en la valoración de la intervención (Soto-Pérez y Franco-Martín, 2014)

Las eSalud han demostrado su utilidad en programas de reducción de daños asociados al consumo de alcohol y tabaco en ensayos clínicos controlados (Chiauzzi, Green, Lord, Thum y Goldstein, 2005; Kypri et al., 2004; Kypri y McAnally, 2005; Neighbors, Larimer y Lewis, 2004; Neighbors, Larimer, Lostutter y Woods, 2006; Walters, Vader

y Harris, 2007). Los beneficios comunes tienen que ver con el anonimato del usuario y con la posibilidad de acceder a los servicios en el momento preciso en que el usuario lo necesita (Marlatt y Witkiewitz, 2010). La eSalud mejora el contacto con los servicios y aumenta la adherencia de personas en situación de exclusión social extrema (Burda,
Haack, Duarte y Alemi, 2012) y ha demostrado su efectividad en la mejora de la evaluación y la prevención de la sobredosis (Baldacchino et al., 2016). Asimismo, WhatsApp ${ }^{\circledR}$ presenta buenos resultados como medio de comunicación rápido y de muy bajo coste, con potencial para mejorar las comunicaciones clínicas y el aprendizaje del paciente sobre su proceso al tiempo que preserva su privacidad (Kamel-Boulos, Giustini y Wheeler, 2016; Nardo et al., 2016; Schreiner y Hess, 2015).

En el uso específico en adicciones, la discusión grupal a través de grupos de WhatsApp ${ }^{\circledR}$ es efectiva para reducir las recaídas gracias a la comunicación directa y rápida y el apoyo social (Cheung et al., 2015) y los profesionales sociales y sanitarios la perciben como potencialmente beneficiosa en la práctica clínica (Ganasegeran, Renganathan, Rashid y Al-Dubai, 2017). A ello se le suman los beneficios generales del uso del móvil en la salud como la posibilidad de transmitir la información de manera eficaz y económica, el acceso a redes sociales de apoyo, todo ello con un componente de inmediatez (Gravenhorst et al., 2015). La inmediatez es un factor común beneficioso de la mSalud en adicciones independientemente de las especificidades del programa (Marlatt y Witkiewitz, 2010). Como vemos este beneficio se potencia en los servicios de mensajería instantánea porque los participantes pueden acceder al apoyo del grupo en cualquier momento y a cualquier hora y pueden obtener respuesta más rápidamente que en los centros de tratamiento especializados, posicionándose como un recurso con muchas potencialidades para retener pacientes en situaciones socioeconómicas significativamente desestructuradas y en exclusión social (La Sala y Mignone, 2014; McInnes, Li y Hogan, 2013). El elevado número de PCDVP entre población sin-hogar en el contexto de la intervención (Calvo-García, Giralt-Vázquez, Calvet-Roura y Carbonell-Sánchez, 2016), la relación entre consumo inyectado crónico y riesgo de sinhogarismo como situación de exclusión social extrema (Des Jarlais, Kerr, Carrieri, Feelemyer y Arasteh, 2016) y el sinhogarismo como nuevo factor de riesgo de infección de VIH entre PCDVP (Folch et al., 2016).

En sí mismo, el uso de teléfonos móviles ha demostrado su efectividad para aumentar la retención y la adherencia terapéutica en adicciones (Ganasegeran et al., 2017; Wolfe, Carrieri y Shepard, 2010). La incorporación de las RSO y de los servicios de mensajería instantánea a las intervenciones virtuales en reducción de daños, respetan los principios de la REDAN (retención, acompañamiento y respetos de los tiempos de la PCDVP en el proceso de la adicción) debido a su carácter de universalidad y a ser una "presencia virtual”. Además, el paciente tiene la posibilidad de usar este tipo de prestaciones virtuales cuando lo necesite y de forma coste-efectiva difícilmente equiparable a otro tipo de intervenciones.

Los resultados obtenidos en este estudio presentan algunas limitaciones que deben ser consideradas. En primer lu- 
gar, el reducido tamaño de la muestra que ha respondido a la necesidad de diseñar y gestionar las sesiones grupales de forma adecuada, teniendo en cuenta las recomendaciones sobre el número máximo de participantes para este tipo de grupos. Sería importante pues replicar el estudio en otros contextos y centros para aumentar así la muestra analizada. En segundo lugar, la RAB todavía no se ha validado en población española. Sería conveniente ampliar el estudio para validar la adaptación realizada al tratarse de un instrumento que permite evaluar los comportamientos de riesgo de infección de VIH asociados a los comportamientos de riesgo de inyección y sexual. Además, el número de observaciones tomadas de la $\mathrm{RAB}$ ha sido limitado y los análisis de consistencia interna, aunque aceptable, han sido inferiores a las de la escala en su versión original. Aun así, para reducir el riesgo de un error sistemático se recurrió a la aleatorización de los participantes y los resultados han demostrado la equivalencia entre los grupos en el momento basal. No obstante, aun teniendo una validez interna aceptable, es necesario ampliar la muestra para limitar la posibilidad de un error aleatorio. En tercer lugar, una observación a seis meses después de la intervención hubiese sido interesante para comprobar si los resultados obtenidos permanecían, disminuían o se extinguían pero las dificultades de acceso a la muestra después de este periodo lo dificultaban. En cuarto lugar, en ausencia de investigaciones similares sobre el uso de WhatsApp ${ }^{\circledR}$ como canal de comunicación para el desarrollo de terapias de reducción de daños grupales, no se han podido comparar los resultados a otros estudios. Finalmente, el acceso a un teléfono móvil y a los servicios como las RSO aún representa una limitación importante para una parte de la población con mayores criterios de exclusión social. En nuestro estudio, no tener teléfono inteligente ha sido motivo de exclusión y por tanto los resultados están circunscritos a aquellos que sí tenían teléfono móvil y usaban habitualmente RSO. En futuros estudios debería revertirse este hecho para evitar los sesgos de participación debidos a esta circunstancia tanto por la tenencia del teléfono como por la habilidad para su uso y manejo.

En conclusión, el uso de las RSO en el campo de las intervenciones de reducción de daños es incipiente y creemos que este trabajo apoya el uso de servicios de mensajería instantánea en el tratamiento virtual. Las RSO presentan múltiples potencialidades para contribuir a la disminución de la exposición al riesgo de infección del VIH, mejorar la retención y aumentar la participación de consumidores de drogas inyectadas.

\section{Conflicto de intereses}

Los autores declaran la ausencia de cualquier tipo de conflicto de intereses.

\section{Referencias}

Armayones, M., Boixadós, M., Gómez, B., Guillamón, N., Hernández, E., Nieto, R.,... Sara, B. (2015). Psicología 2.0: Oportunidades y retos para el profesional de la psicología en el ámbito de la salud. Papeles del Psicólogo, 36, 153-160.

Baldacchino, A., Crocamo, C., Humphris, G., Neufeind, J., Frisher, M., Scherbaum, N. y Carrà, G. (2016). Decision support in addiction: The development of an e-health tool to assess and prevent risk of fatal overdose. The ORION Project. Computer Methods and Programs in Biomedicine, 133, 207-216. doi:10.1016/j.cmpb.2016.05.018.

Barrio, G., Bravo, M. J., Brugal, M. T., Díez, M., Regidor, E., Belza, M. J. y de la Fuente, L. (2012). Harm reduction interventions for drug injectors or heroin users in Spain: Expanding coverage as the storm abates. Addiction, 107, 1111-1122. doi:10.1111/j.1360-0443.2011.03759.x.

Bosque-Prous, M. y Brugal, M. T. (2016). Intervenciones de reducción de daños en usuarios de drogas: Situación actual y recomendaciones. Gaceta Sanitaria, 30, 99-105. doi:10.1016/j.gaceta.2016.04.020.

Burda, C., Haack, M., Duarte, A. C. y Alemi, F. (2012). Medication adherence among homeless patients: A pilot study of cell phone effectiveness. Journal of the American Academy of Nurse Practitioners, 24, 675-681. doi:10.1111/ j.1745-7599.2012.00756.x.

Calvo-García, F., Giralt-Vázquez, C., Calvet-Roura, A. y Carbonell-Sánchez, X. (2016). Riesgo de suicidio en población sin hogar. Clínica y Salud, 27, 89-96. doi:10.1016/j. clysa.2016.05.002.

Calvo-García, F., Turró-Garriga, O. y Giralt-Vázquez, C. (2014). El consumo activo de drogas de pacientes incluidos en un programa de tratamiento de mantenimiento con metadona. Revista de Trabajo Social y Salud, 79, $57-68$.

Calvo, P., Pérez, A., Sacristán, P. y Paricio, C. (2009). Terapia grupal en prevención de recaídas del alcoholismo frente a seguimiento ambulatorio habitual. Adicciones, 21, 15-20. doi:10.20882/adicciones.247.

Calvo F. y Carbonell, X. (2018). Using Facebook for improving the psychological well- being of individuals experiencing homelessness: Experimental and longitudinal study. JMIR Mental Health, 5, e59. doi:10.2196/ mental.9814.

Calvo, F., Carbonell, X. y Badia, M. (2018). Homelessness and unemployment during the economic recession: The case of the city of Girona. European Scientific Journal, 14, 1857-7881. doi:10.19044/esj.2018.v14n13p59.

Calvo, F., Carbonell, X., Giralt, C., Lloberas, À. y Turró, O. (2017). Reducción de daños asociados al consumo inyectado de drogas en población sin-hogar: Propuesta para una intervención grupal a través de WhatsApp. Pedagogia i Treball Social, 6, 3-31. 
Calvo, F., Carbonell, X., Turró, O. y Giralt, C. (2018). Connected in the street: The relation between online social networks, selfesteem and satisfaction with life among individuals experiencing homelessness. Aloma, 36, 21-28.

Calvo, F., Carbonell, X., Valero, R., Costa, J., Turró, O., Giralt, C. y Ramírez, M. (2018). Abandono precoz y retención en servicios ambulatorios de drogodependencias: Análisis transversal comparativo de factores que aumentan o disminuyen la adherencia. Atención Primaria, 50, 477-485. doi:10.1016/j.aprim.2017.06.006.

Cheung, Y. T., Chan, C. H., Lai, C.K, Chan, W. F., Wang, M. P., Li, H. C.,... Lam, T.H. (2015). Using WhatsApp and Facebook online social groups for smoking relapse prevention for recent quitters: A pilot pragmatic cluster randomized controlled trial. Journal of Medical Internet Research, 17, e238. doi:10.2196/jmir.4829.

Chiauzzi, E., Green, T. C., Lord, S., Thum, C. y Goldstein, M. (2005). My student body: A high-risk drinking prevention web site for college students. Journal of American College Health, 53, 263-274. doi:10.3200/JACH.53.6.263274.

Des Jarlais, D. C., Kerr, T., Carrieri, P., Feelemyer, J. y Arasteh, K. (2016). HIV infection among persons who inject drugs: Ending old epidemics and addressing new outbreaks. AIDS, 30, 815-826. doi:10.1097/ QAD.0000000000001039.

Erickson, P. (1995). Harm reduction: What it is and is not. Drug and Alcohol Review, 14, 283-285. doi:10.1080/09595239500185361.

Fernández, J. J., González, M. P., Saiz, P. A., Gutiérrez, E. y Bobes, J. (1999). Calidad de vida y severidad de la adicción en heroinómanos en mantenimiento prolongado con metadona. Adicciones, 11, 43. doi:10.20882/adicciones.594.

Folch, C., Casabona, J., Espelt, A., Majó, X., Meroño, M., Gonzalez, V.,... Group, R. S. (2016). High prevalence and incidence of HIV and HCV among new injecting drug users with a large proportion of migrants. Is prevention failing? Substance Use E Misuse, 51, 250-260. doi:10.3109/10826084.2015.1092991.

Fuente, L., Brugal, T., Domingo-Salvany, A., Bravo, M. J., Neira-León, M. y Barrio, G. (2006). Más de treinta años de drogas ilegales en España: Una amarga historia con algunos consejos para el futuro. Revista Española de Salud Pública, 80, 505-520.

Ganasegeran, K., Renganathan, P., Rashid, A. y Al-Dubai, S. A. R. (2017). The mhealth revolution: Exploring perceived benefits of WhatsApp use in clinical practice. International Journal of Medical Informatics, 97, 145-151. doi:10.1016/j.ijmedinf.2016.10.013.

Genz, A., Kirk, G., Piggott, D., Mehta, S. H., Linas, B. S. y Westergaard, R. P. (2015). Uptake and acceptability of information and iommunication technology in a community-based cohort of people who inject drugs: Impli- cations for mobile health interventions. JMIR MHealth and UHealth, 3, e70. doi.org/10.2196/mhealth.3437.

Gravenhorst, F., Muaremi, A., Bardram, J., Grünerbl, A., Mayora, O., Wurzer, G.,... Tröster, G. (2015). Mobile phones as medical devices in mental disorder treatment: An overview. Personal and Ubiquitous Computing, 19, 335-353. doi:10.1007/s00779-014-0829-5.

Kamel-Boulos, M., Giustini, D. y Wheeler, S. (2016). Instagram and WhatsApp in health and healthcare: An overview. Future Internet, 8, 37. doi:10.3390/fi8030037.

Kaplan, A. M. y Haenlein, M. (2010). Users of the world, unite! The challenges and opportunities of social media. Business Horizons, 53, 59-68. doi:10.1016/j.bushor.2009.09.003.

Kypri, K. y McAnally, H. M. (2005). Randomized controlled trial of a web-based primary care intervention for multiple health risk behaviors. Preventive Medicine, 41, 761-766. doi:10.1016/J.YPMED.2005.07.010.

Kypri, K., Saunders, J. B., Williams, S. M., McGee, R. O., Langley, J. D., Cashell-Smith, M. L. y Gallagher, S. J. (2004). Web-based screening and brief intervention for hazardous drinking: A double-blind randomized controlled trial. Addiction, 99, 1410-1417. doi:10.1111/ j.1360-0443.2004.00847.x.

La Sala, A. y Mignone, J. (2014). The benefits of information communication technology use by the homeless: A narrative synthesis review. Journal of Social Distress and the Homeless, 23, 51-67. doi:10.1179/1573658X1 4Y.0000000006.

Little, J., Hodari, K., Lavender, J. y Berg, A. (2008). Come as you are: Harm reduction drop-in groups for multidiagnosed drug users. Journal of Groups in Addiction $\mathcal{E}^{\circ}$ Recovery, 3, 161-192. doi:10.1080/15560350802424845.

Lozano, R., Domeque, N., Perálvarez, C., Torrellas, M. D. y Gonzalo, C. (2019). Mortality rate in patients on methadone treatment and infected with the human immunodeficiency virus and/or the hepatitis $\mathrm{C}$ virus. Adicciones, 31, 78-79. doi:10.20882/adicciones.1007.

Marlatt, G. A. y Witkiewitz, K. (2010). Update on harm-reduction policy and intervention research. Annual Review of Clinical Psychology, 6, 591-606. doi:10.1146/annurev. clinpsy.121208.131438.

Martínez-González, J., Albein-Urios, N., Lozano-Rojas, O. y Verdejo-García, A. (2014). Aspectos diferenciales de riesgo de abandono al inicio del tratamiento de la adicción a la cocaína en pacientes con trastorno de personalidad. Adicciones, 26, 116-125. doi:10.20882/adicciones.13.

Martínez-Luna, N. G., Rodríguez-Cintas, L., Esojo, A., Palma-Álvarez, R. F., Robles-Martínez, M., Grau-López, L., ... Roncero, C. (2018). Harm reduction program use, psychopathology and medical severity in patients with methadone maintenance treatment. Adicciones, 30, 197207. doi:10.20882/adicciones.897. 
McInnes, D. K., Li, A. E. y Hogan, T. P. (2013). Opportunities for engaging low-income, vulnerable populations in health care: A systematic review of homeless persons' access to and use of information technologies. American Journal of Public Health, 103, 11-20.

Mira, J. J., Llinás, G., Lorenzo, S. y Aibar, C. (2009). Uso de internet por médicos de primaria y hospitales y percepción de cómo influye en su relación con los pacientes. Atención Primaria, 41, 308-314. doi:10.1016/j. aprim.2008.10.007.

Nardo, B., Cannistrà, M., Diaco, V., Naso, A., Novello, M., Zullo, A.,... Sacco, R. (2016). Optimizing patient surgical management using WhatsApp application in the italian healthcare system. Telemedicine and E-Health, 22, 718-725. doi:10.1089/tmj.2015.0219.

National Institute on Drug Abuse. (2018). Risk Assessment Battery |Data Share 2.0. Recuperado de https:/ / datashare. nida.nih.gov/instrument/risk-assessment-battery.

Navaline, H. A., Snider, E. C., Petro, C. J., Tobin, D., Metzger, D., Alterman, A. I. y Woody, G. E. (1994). Preparations for AIDS vaccine trials. An automated version of the risk assessment battery (RAB): Enhancing the assessment of risk behaviors. AIDS Research and Human Retroviruses, 10, S281-S283.

Neighbors, C., Larimer, M. E. y Lewis, M. A. (2004). Targeting misperceptions of descriptive drinking norms: Efficacy of a computer-delivered personalized normative feedback intervention. Journal of Consulting and Clinical Psychology, 72, 434-447.

Neighbors, C., Larimer, M. E., Lostutter, T. W. y Woods, B. A. (2006). Harm reduction and individually focused alcohol prevention. International Journal of Drug Policy, 17, 304-309. doi:10.1016/J.DRUGPO.2006.05.004.

Rice, E. (2010). The positive role of social networks and social networking technology in the condom-using behaviors of homeless young people. Public Health Reports, 125, 588-595.

Rice, E., Milburn, N. G. y Monro, W. (2011). Social networking technology, social network composition, and reductions in substance use among homeless adolescents. Prevention Science, 12, 80-88. doi:10.1007/ s11121-010-0191-4.

Rice, E., Tulbert, E., Cederbaum, J., Barman-Adhikari, A. y Milburn, N. G. (2012). Mobilizing homeless youth for HIV prevention: A social network analysis of the acceptability of a face-to-face and online social networking intervention. Health Education Research, 27, 226-236. doi:10.1093/her/cyr113.

Schreiner, M. y Hess, T. (2015). Examining the role of privacy in virtual migration: the case of WhatsApp and Threema. AIS Electronic Library (Vol. 33). München: Universität München.

Soto-Pérez, F. y Franco-Martín, M. (2014). PsicoED: Una alternativa online y comunitaria para la psicoeduca- ción en esquizofrenia. Psicoperspectivas, 13, 118-129. doi: 10.5027 / PSICOPERSPECTIVAS-VOL13-ISSUE3-FULLTEXT-416.

Stone, K. (2014). The global state of harm reduction 2014. London. Recuperado de https://www.hri.global/files/2015/02/16/GSHR2014.pdf.

Stone, K. (2016). The global state of harm reduction 2016. London: Harm Reduction International. Recuperado de https:/ /www.hri.global/files/2016/11/14/GSHR2016_ 14nov.pdf.

Timko, C., Schultz, N. R., Cucciare, M. A., Vittorio, L. y Garrison-Diehn, C. (2016). Retention in medication-assisted treatment for opiate dependence: A systematic review. Journal of Addictive Diseases, 35, 22-35. doi:10.1080/ 10550887.2016.1100960.

Trujols, J., Iraurgi, I., Solà, I., Ballesteros, J., Siñol, N., Batlle, F. y de los Cobos, J. P. (2010). Infección por VIH y usuarios de drogas por vía parenteral: Urgencia de la revitalización de los programas de reducción de daños. Adicciones, 22, 135-140. doi:10.20882/adicciones.202.

Walters, S. T., Vader, A. M. y Harris, T. R. (2007). A controlled trial of web-based feedback for heavy drinking college students. Prevention Science, 8, 83-88. doi:10.1007/ s11121-006-0059-9.

World Health Organization. (2011). mHealth. New horizons for heath through mobile technologies. Geneva: WHO. Recuperado de https://www.who.int/goe/publications/ goe_mhealth_web.pdf.

Wolfe, D., Carrieri, M. P. y Shepard, D. (2010). Treatment and care for injecting drug users with HIV infection: A review of barriers and ways forward. Lancet, 376, 355366. doi:10.1016/S0140-6736(10)60832-X. 
$\square$ Marcar en caso de que sea el entrevistador que lea las preguntas.

ID\#:

FECHA

Administrada por:

Corregida por:

\section{Escala de Valoración de Riesgo (RISK AsSessment BATTERY)}

\section{R A B}

Por favor, lea detenidamente cada una de las siguientes preguntas. Como puede observar, muchas de ellas son de carácter personal. Entendemos lo importante que esto es para usted su privacidad, por lo tanto, haremos todos los esfuerzos para garantizar la confidencialidad de sus respuestas.

Es muy importante que sus respuestas sean honestas. Es mejor que no responda a una pregunta a que nos dé una respuesta incompleta no del todo cierta. Algunas preguntas quizás no parezcan tener una respuesta del todo exacta para usted. En esos casos, responda la que más se aproxime a su situación. No dedique demasiado tiempo a cada respuesta. Recuerde solicitar ayuda siempre que lo necesite.

Gracias por su tiempo y su cooperación.

\section{USO DE ALCOHOL Y OTRAS DROGAS EL MES PASADO}

A. El mes pasado, ¿con qué frecuencia se inyectó cocaína y heroína juntas (Speedball)?
$0 . \square$ Nunca
1. $\square$ Algunas veces
2. $\square$ Algunas veces cada semana
3. $\square$ A diario

B. El mes pasado, ¿con qué frecuencia se inyectó heroína (sin incluir Speedball)?
0 . $\square$ Nunca
1. $\square$ Algunas veces
2. $\square$ Algunas veces cada semana
3. $\square$ A diario

C. El mes pasado, ¿con qué frecuencia esnifó heroína (sin incluir Speedball)?
0 . $\square$ Nunca
1. $\square$ Algunas veces
2. $\square$ Algunas veces cada semana
3. $\square$ A diario

D. El mes pasado, ¿con qué frecuencia fumó heroína?
$0 . \square$ Nunca
1. $\square$ Algunas veces
2. $\square$ Algunas veces cada semana
3. $\square$ A diario

E. El mes pasado, ¿con qué frecuencia se inyectó cocaína (sin incluir Speedball)?
0 . $\square$ Nunca
1. $\square$ Algunas veces
2. $\square$ Algunas veces cada semana
3. $\square$ A diario

F. El mes pasado, ¿con qué frecuencia esnifó cocaína (sin incluir Speedball)?
0 . $\square$ Nunca
1. $\square$ Algunas veces
2. $\square$ Algunas veces cada semana
3. $\square$ A diario 
G. El mes pasado, ¿con qué frecuencia fumó crack, pasta base, base o cocaína?
$0 . \square$ Nunca
1. $\square$ Algunas veces
2. $\square$ Algunas veces cada semana
3. $\square$ A diario

H. El mes pasado, ¿con qué frecuencia se inyectó anfetaminas, meta, meth, cristal, speed o crank?
$0 . \square$ Nunca
1. $\square$ Algunas veces
2. $\square$ Algunas veces cada semana
3. $\square$ A diario

I. El mes pasado, ¿con qué frecuencia esnifó anfetaminas, meta, meth, cristal, speed o crank?
$0 . \square$ Nunca
1. $\square$ Algunas veces
2. $\square$ Algunas veces cada semana
3. $\square$ A diario

J. El mes pasado, ¿con qué frecuencia fumó anfetaminas, meta, meth, cristal, speed o crank?
0. $\square$ Nunca
1. $\square$ Algunas veces
2. $\square$ Algunas veces cada semana
3. $\square$ A diario

K. El mes pasado, ¿con qué frecuencia usó benzodiazepinas (benzos) como Xanax, Valium, Diazepan, Tetrazepam, Lorazepam, Trankimazin, Aprazolam
0. $\square$ Nunca
1. $\square$ Algunas veces
2. $\square$ Algunas veces cada semana
3. $\square$ A diario

L. El mes pasado, ¿con qué frecuencia tomó analgésicos? Píldoras como Tramadol, Percocet, Vicodin, Demerol, Dilaudid, Darvon, Darvocet o Codeina
$0 . \square$ Nunca
1. $\square$ Algunas veces
2. $\square$ Algunas veces cada semana
3. $\square$ A diario
a. Qué analgésicos usa?

M. El mes pasado, ¿con qué frecuencia se inyectó Dilaudid, Metadona u otra medicación derivada del opio?
0 . $\square$ Nunca
1. $\square$ Algunas veces
2. $\square$ Algunas veces cada semana
3. $\square$ A diario

N. El mes pasado, ¿con qué frecuencia utilizó ácido lisérgico, LSD, setas, hongos cactus u otros alucinógenos?
0 . $\square$ Nunca
1. $\square$ Algunas veces
2. $\square$ Algunas veces cada semana
3. $\square$ A diario

O. El mes pasado, ¿con qué frecuencia utilizó marihuana, hachís u otro derivado del cannabis?
0 . $\square$ Nunca
1. $\square$ Algunas veces
2. $\square$ Algunas veces cada semana
3. $\square$ A diario

P. El mes pasado, ¿con qué frecuencia bebió cerveza, vino o cualquier otra bebida alcohólica?
$0 . \square$ Nunca
1. $\square$ Algunas veces
2. $\square$ Algunas veces cada semana
3. $\square$ A diario 


\section{PARTE I: USO DE MATERIAL DE INYECCIÓN}

1. ¿Se ha inyectado drogas durante los últimos 6 meses?
$0 . \square$ No
1. $\square$ Sí

2. ¿Ha compartido jeringuillas o algún material de inyección (cazoletas u otros recipientes, filtro, algodón, etcétera durante los últimos 6 meses?

0 . $\square$ No o no me he inyectado los últimos 6 meses

3. $\square$ Sí

3. ¿Con cuánta gente diferente ha compartido jeringuillas los últimos 6 meses?

0 . $\square$ Ninguna o no me he inyectado los últimos 6 meses

1. $\square 1$ persona diferente

2. $\square 203$ personas diferentes

3. $\square 40$ más personas diferentes

4. ¿Con qué frecuencia ha usado una jeringuilla usada por otra persona los últimos 6 meses (lavada o sin lavar)?

0 . $\square$ Nunca o no me he inyectado los últimos 6 meses

1. $\square$ Algunas veces

2. $\square$ Algunas veces cada mes

3. $\square$ Una vez por semana o más veces

5. ¿Con qué frecuencia otras personas han utilizado una jeringuilla usada por usted los últimos 6 meses (lavada o sin lavar)?

0. $\square$ Nunca o no me he inyectado los últimos 6 meses

1. $\square$ Algunas veces

2. $\square$ Algunas veces cada mes

3. $\square$ Una vez por semana o más veces

6. Los últimos 6 meses, ¿Con qué frecuencia ha compartido jeringuillas con alguien que sabía (o te enteró después) que tuviera SIDA, o fuera seropositivo (infectado de VIH, el virus del SIDA)?

0 . $\square$ Nunca o no me he inyectado los últimos 6 meses

1. $\square$ Algunas veces

2. $\square$ Algunas veces cada mes

3. $\square$ Una vez por semana o más veces

7. ¿Dónde consiguió sus jeringuillas durante los últimos 6 meses? (Puedes marcar varias respuestas si es necesario)

0 . $\square$ No me he inyectado los últimos 6 meses.

1. $\square$ De una persona diabética

2. $\square$ Las he cogido en la calle

3. $\square$ Me las dio o vendió el traficante

4. $\square$ En un lugar donde otras personas van a inyectarse (chutadero, casa ocupada, etc.)

5. $\square$ Punto de Intercambio de Jeringuillas (CAS, ambulatorio, hospital, etc.)

6. $\square$ Otros
8. En los ultimo 6 meses, ¿con qué frecuencia ha estado en un chutadero, casa ocupada u otro lugar donde la gente acostumbra a ir para inyectarse drogas?

0 . $\square$ Nunca

1. $\square$ Algunas veces

2. $\square$ Algunas veces cada mes

3. $\square$ Una vez por semana o más veces

9. En los últimos 6 meses, ¿con qué frecuencia ha estado en un fumadero $u$ otro lugar donde la gente acostumbra $a$ ir para fumar drogas?
0 . $\square$ Nunca
1. $\square$ Algunas veces
2. $\square$ Algunas veces cada mes
3. $\square$ Una vez por semana o más veces

10. ¿Qué frase describe mejor la manera de limpiar las jeringuillas que tuvo durante los últimos 6 meses? (Por favor, escoja solo una opción)

0. $\square$ No me he inyectado en los últimos 6 meses

1. $\square$ Siempre utilizo jeringuillas nuevas

2. $\square$ Siempre limpio las jeringuillas justo antes de inyectarme

3. $\square$ Siempre limpio las jeringuillas justo después de inyectarme

4. $\square$ A veces limpio las jeringuillas, a veces no

5. $\square$ Nunca limpio las jeringuillas

11. Si limpió sus jeringuillas u otros materiales de inyección como cazoletas, recipientes, etc, los últimos 6 meses ¿cómo lo hizo? (puede marcar varias opciones)

0 . $\square$ No me he inyectado en los últimos 6 meses

1. $\square$ Agua y jabón o agua solamente

2. $\square$ Alcohol

3. $\square$ Lejía

4. $\square$ Agua hirviendo

5. $\square$ Otras:

6. $\square$ No he limpiado mis jeringuillas los últimos 6 meses

7. $\square$ Yo siempre he usado jeringuillas nuevas los últimos 6 meses

12. ¿Con qué frecuencia ha compartido agua estéril (el agua que viene en el kit de intercambio de jeringuillas) durante los últimos 6 meses?

0 . $\square$ Nunca o no me he inyectado los últimos 6 meses

1. $\square$ Algunas veces

2. $\square$ Algunas veces cada mes

3. $\square$ Una vez por semana o más veces 
13. ¿Con qué frecuencia ha compartido cazoletas u otro recipiente para preparar las dosis, durante los últimos 6 meses?

0 . $\square$ Nunca o no me he inyectado los últimos 6 meses

1. $\square$ Algunas veces

2. $\square$ Algunas veces cada mes

3. $\square$ Una vez por semana o más veces

14. ¿Con qué frecuencia ha compartido filtros o algodones para filtrar las dosis, durante los últimos 6 meses?

0. $\square$ Nunca o no me he inyectado los últimos 6 meses

1. $\square$ Algunas veces

2. $\square$ Algunas veces cada mes

3. $\square$ Una vez por semana o más veces
15. En los últimos 6 meses, ¿con qué frecuencia ha dividido dosis con otras personas usando una jeringuilla para introducir o cargar a/desde otra jeringuilla dicha dosis (backfrontloading, por ejemplo)?

0 . $\square$ Nunca o no me he inyectado los últimos 6 meses

1. $\square$ Algunas veces

2. $\square$ Algunas veces cada mes

3. $\square$ Una vez por semana o más veces

\section{PARTE II: PRACTICAS SEXUALES}

16. ¿Cómo se describiría a usted mismo?
1. $\square$ Heterosexual
2. $\square$ Gay/Homosexual/Lesbiana
3. $\square$ Bisexual

Atención: Para las siguientes preguntas, consideraremos "sexo" como cualquier relación sexual con penetración (vaginal o anal) o sexo oral (felación, cunnilingus, por ejemplo).

17. ¿Con cuántos hombres ha tenido relaciones sexuales durante los últimos 6 meses?
0 . $\square 0$ hombres
1. $\square 1$ hombre
2. $\square 203$ hombres
3. $\square 40$ más hombres

18. ¿Con cuantas mujeres ha tenido relaciones sexuales durante los últimos 6 meses?
0. $\square 0$ mujeres
1. $\square 1$ mujer
2. $\square 2$ o 3 mujeres
3. $\square 40$ más mujeres

19. En los últimos 6 meses, ¿con qué frecuencia ha mantenido relaciones sexuales para conseguir drogas?
$0 . \square$ Nunca
1. $\square$ Algunas veces
2. $\square$ Algunas veces cada mes
3. $\square$ Una vez por semana o más veces

20. En los últimos 6 meses, ¿con qué frecuencia le ha dado drogas a otra persona para poder tener sexo con él/ella?

$0 . \square$ Nunca

1. $\square$ Algunas veces

2. $\square$ Algunas veces cada mes

3. $\square$ Una vez por semana o más veces
21. En los últimos 6 meses, ¿Con qué frecuencia pagó dinero para tener sexo con alguien?
0 . $\square$ Nunca
1. $\square$ Algunas veces
2. $\square$ Algunas veces cada mes
3. $\square$ Una vez por semana o más veces

22. En los últimos 6 meses, ¿con qué frecuencia le dio dinero a alguien con el objetivo de tener sexo con ellos/ellas?
0 . $\square$ Nunca
1. $\square$ Algunas veces
2. $\square$ Algunas veces cada mes
3. $\square$ Una vez por semana o más veces

23. En los últimos 6 meses, ¿con qué frecuencia ha tenido relaciones sexuales con alguien que sabía (antes o después de tener estas relaciones) que tenía SIDA o era seropositivo (VIH positivo, la infección del SIDA)?
0 . $\square$ Nunca
1. $\square$ Algunas veces
2. $\square$ Algunas veces cada mes
3. $\square$ Una vez por semana o más veces

24. En los últimos 6 meses, ¿con qué frecuencia ha usado preservativo para mantener relaciones sexuales?

0 . $\square$ No he mantenido relaciones sexuales los últimos 6 meses.

1. $\square$ Todas las veces

2. $\square$ La mayor parte de las veces

3. $\square$ Algunas veces

4. $\square$ Nunca 


\section{PARTE III: SOBRE VIH Y PRUEBAS RELACIONADAS}

Si ya sabes que eres VIH positivo, pasa a la pregunta \#28.

25. ¿Cuál es su nivel de preocupación ante la posibilidad de estar infectado de VIH o padecer SIDA?
0 . $\square$ Nada
1. $\square$ Ligeramente
2. $\square$ Moderadamente
3. $\square$ Considerablemente
4. $\square$ Extremadamente

26. ¿Cuál es su nivel de preocupación ante la posibilidad de haber estado expuesto a la infección del VIH/SIDA?
0. $\square$ Nada
1. $\square$ Ligeramente
2. $\square$ Moderadamente
3. $\square$ Considerablemente
4. $\square$ Extremadamente

27. ¿Cuántas veces ha realizado un test de sangre para determinar infección de VIH/SIDA? (HIV)? (indíquelo con un círculo):

$\begin{array}{lllllllllll}0 & 1 & 2 & 3 & 4 & 5 & 6 & 7 & 8 & 9 & 10\end{array}$ o más veces
28. ¿Cuándo se realizó el ultimo test de VIH? En las siguientes líneas indique el mes y el año en que realizó la prueba más reciente.

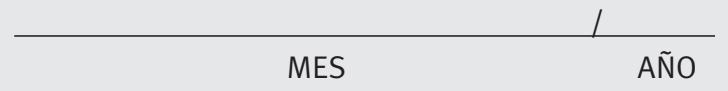

29. ¿Alguna vez le han comunicado que usted está infectado de VIH o padece SIDA?
$0 . \square$ No
1. $\square$ Sí
2. $\square$ Nunca obtuve los resultados 
